\title{
$\mathrm{SKY}$ : 基于分布式 Kautz 图的高效 P2P 网络
}

\author{
张一鸣*, 卢锡城, 李东升 \\ 国防科技大学并行与分布处理国家重点实验室, 长沙 410073 \\ *通信作者 E-mail: ymzhang@nudt.edu.cn
}

收稿日期: 2007-08-22; 接受日期: 2008-02-27

国家自然科学基金 (批准号: 60673167,60703072)、湖南省自然科学基金 (批准号: 08JJ3125) 和国家重点基础研究发展计划 (批准号: 2005CB321801) 资助项目

\begin{tabular}{|c|c|}
\hline 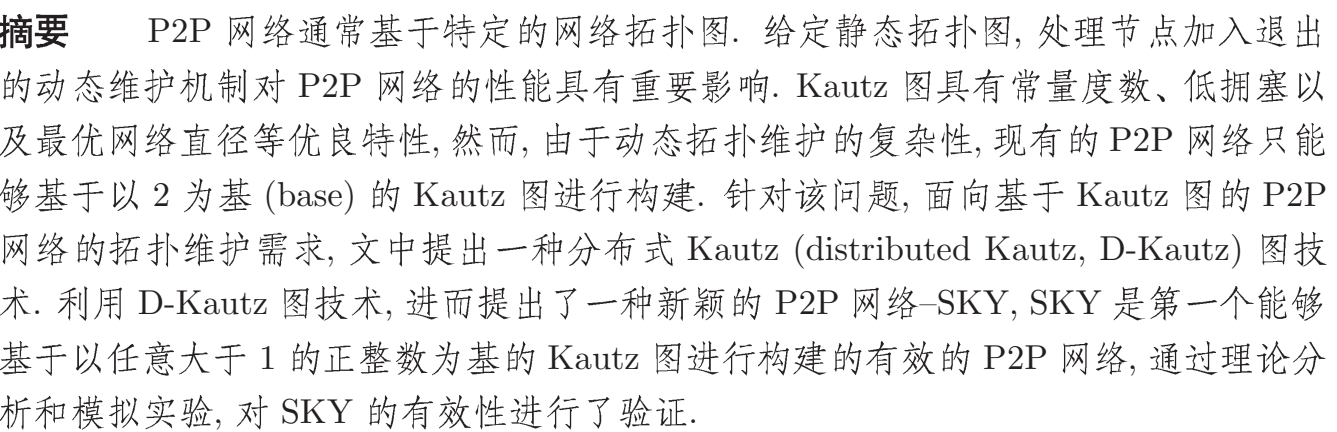 & $\begin{array}{l}\text { 关键词 } \\
\text { peer-to-peer 网络 } \\
\text { Kautz 图 } \\
\text { 常量度数 } \\
\text { 拓扑维护 } \\
\text { D-Kautz 变换 }\end{array}$ \\
\hline
\end{tabular}

\section{0 引言}

近年来, 结构化 peer-to-peer (P2P) 网络在 Internet 中的应用越来越广泛, 例如文件共享、分布 式存储、分布式域名服务、应用层组播和协作式缓 存等 $[1 \sim 6]$. P2P 网络通常基于特定的网络拓扑图进 行构建, 这些拓扑图对系统的网络直径、平均度数以 及负载平衡等性能有重要影响. 很多传统的网络拓 扑图已经被用来设计各种高效的 $\mathrm{P} 2 \mathrm{P}$ 网络. 例如, Chord $^{[7]}$ 基于环形 (ring) 拓扑, Pastry ${ }^{[8]}$ 、 Tapestry ${ }^{[9]}$ 和 Kademlia ${ }^{[10]}$ 基于超立方体 (hypercube) 拓扑, SkipNet ${ }^{[11]}$ 基于跳表 (skip-list) 拓扑, $\mathrm{CAN}^{[12]}$ 基于 d-torus 拓扑, Viceroy ${ }^{[13]}$ 和 Ulysses ${ }^{[14]}$ 基于蝴蝶 (butterfly) 拓扑, Cycloid ${ }^{[15]}$ 基于 Cube-Connected-Cycle 拓扑, D2B ${ }^{[16]} 、$ Koorde $^{[17]} 、$ ODRI ${ }^{[18]}$ 和 Broose ${ }^{[19]}$ 基 于 de Bruijn 图, FissionE ${ }^{[20]}$ 和 Moore ${ }^{[21]}$ 基于 Kautz 图.

P2P 网络性能评价的重要参数有节点度数、网络
直径和拥塞特性等. Kautz 图具有常量度数 (constant degree)、最优网络直径 (optimal diameter)、常量拥 塞 (constant congestion)、高连通度以及简单路由等 优良特性, 近年来在工业界和学术界得到了研究者 的广泛关注. 但是由于处理节点动态加入退出的拓 扑维护机制的复杂性, 目前的 $\mathrm{P} 2 \mathrm{P}$ 网络只能够基于 以 2 为基 (base) 的 Kautz 图进行构建.

动态拓扑维护是 $\mathrm{P} 2 \mathrm{P}$ 网络中的关键技术, 直接 决定着 $\mathrm{P} 2 \mathrm{P}$ 网络及上层应用的性能和可扩展性等. $\mathrm{P} 2 \mathrm{P}$ 网络所面临的难点之一是动态维护机制能够在 任意节点加入退出情况下有效地保持静态拓扑图的 优良特性. 为了解决基于 Kautz 图的 P2P 网络的动 态维护问题, 本文基于线图技术的 “边点变换” 的思 想 ${ }^{[22,23]}$ 和 Kautz 图的特点, 提出一种分布式 Kautz (distributed Kautz, D-Kautz) 图技术. 利用 D-Kautz 图技术, 本文进而提出了一种高效的 P2P 网络 SKY. SKY 是第一个能够基于以任意正整数 $(>2)$ 为基的 Kautz 图进行构建的有效的 P2P 网络. 模 
拟实验结果表明, 与其他常量度数的 P2P 网络 (如 CAN 和 FissionE) 相比, SKY 具有更好的性能.

本文的贡献包括如下 3 个方面.

首先, 我们提出了一种分布式 Kautz 图技术. 使 用该技术可以基于静态 Kautz 图 $K(d, D)$ 获得一系 列 P2P 网络拓扑.

其次, 我们提出了一种高效的 P2P 网络 SKY, 并为 SKY 设计了相应的资源发布, 查找以及路由的 方法. SKY 是第一个能够基于以任意常数为基的 Kautz 图进行构建的有效的 P2P 网络.

最后, 我们通过理论分析和大量模拟实验对 SKY 的正确性进行了验证, 并将 SKY 与其他常量 度数拓扑的 $\mathrm{P} 2 \mathrm{P}$ 网络 (如 CAN 和 FissionE) 进行了 比较.

本文剩余部分的组织如下. 第 1 节对 Kautz 图 的基本概念进行概述. 第 2 节提出分布式 Kautz 图 技术. 第 3 节应用分布式 Kautz 图提出一种新颖的 P2P 网络 SKY. 第 4 节对 SKY 的特性进行模拟 验证. 第 5 节介绍相关工作. 第 6 节总结全文.

\section{1 背景介绍}

本节将简要介绍 Kautz 图的基本定义以及相关 的基本概念. 对字符串 $\xi=a_{1} a_{2} \cdots a_{D}$, 若 $a_{i} \in$ $\{0,1,2, \ldots, d\}(1 \leqslant i \leqslant D)$ 且 $a_{i} \neq a_{i+1}(1 \leqslant i \leqslant$ $D-1)$, 则称 $\xi$ 是基为 $d$, 长度为 $D$ 的 Kautz 串. Kautz 空间 $\operatorname{KautzSpace}(d, D)$ 是指所有长度为 $D$, 基 为 $d$ 的 Kautz 串的集合.

Kautz 图 $K(d, D)$ 是一个有向图, 其中每个节点 的标识都是 Kautz 空间 KautzSpace $(d, D)$ 中的一个 Kautz 串. $K(d, D)$ 中每个节点 $u=u_{1} u_{2} \cdots u_{D}$ 都有 $d$ 条出边: 对任意 $\alpha \in\{0,1,2, \cdots, d\}$ 且 $\alpha \neq u_{D}, u$ 有一条到 $v=u_{2} u_{3} \cdots u_{D} \alpha$ 的出边 (用 $[u, v]$ 或 $u \rightarrow v$ 来表示). 图 1 给出了两个 Kautz 图示例 $K(2,1)$ 和 $K(2,2)$.

设 $u_{D} \neq v_{1}$, 从节点 $u=u_{1} u_{2} \cdots u_{D}$ 到 $v=$ $v_{1} v_{2} \cdots v_{D}$ 的路由过程如下:

$$
\begin{array}{r}
u=u_{1} u_{2} \cdots u_{D} \rightarrow u_{2} \cdots u_{D} v_{1} \rightarrow \cdots \rightarrow \\
u_{D} v_{1} v_{2} \cdots v_{D-1} \rightarrow v_{1} v_{2} \cdots v_{D}=v .
\end{array}
$$

在 Kaut 图中, $|u|$ 表示节点 $u$ 的标识长度, 例如
若 $u=u_{1} u_{2} \cdots u_{k}$, 则 $|u|=k$. 假设 $a=[x, y]$ 为从点 $x$ 到点 $y$ 的一条边, 我们称: $a$ 为 $y$ 的入边, $y$ 为 $a$ 的 终点; $a$ 为 $x$ 的出边, $x$ 为 $a$ 的起点; $x$ 为 $y$ 的入边 邻居 (入点), $y$ 为 $x$ 的出边邻居 (出点). 令 $\Gamma_{G}^{+}(x)$ 和 $\Gamma_{G}^{-}(x)$ 分别为点 $x$ 在图 $G$ 中的出边邻居集合与入边 邻居集合. 节点 $x$ 的出度 (out-degree) 是指 $x$ 的出 边邻居个数, 即 $\delta_{G}^{+}(x)=\left|\Gamma_{G}^{+}(x)\right|$, 节点 $x$ 的入度 (indegree) 是指 $x$ 的入边邻居个数, 即 $\delta_{G}^{-}(x)=\left|\Gamma_{G}^{-}(x)\right|$. 如果图 $G$ 中的任意节点 $x$ 都具有相同的出度, 我们 称 $G$ 为常量出度 (constant out-degree) 图; 如果图 $G$ 中的任意节点 $x$ 都具有相同的入度, 我们称 $G$ 为常 量入度 (constant in-degree) 图. 如果一个图既是常 量出度图又是常量入度图, 那么我们称它为常量度数 (constant degree) 图. 显然, Kautz 图是常量度数图.

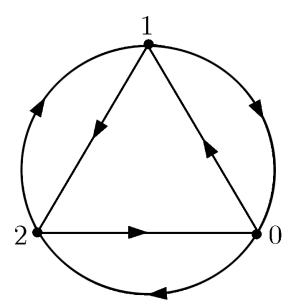

(a)

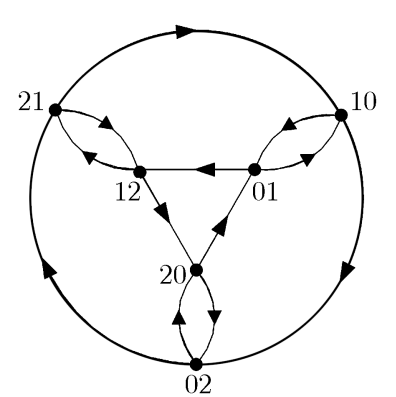

(b)
图 1 Kautz 图示例 ${ }^{[22]}$

(a) $K(2,1) ;($ b) $K(2,2)$

文献 [22] 指出, 给定节点数 $N$ 和最大出度 $d$, Kautz 图 $K(d, D)$ 是网络直径 $D$ 最小的拓扑图; 给 定节点度数 $d$ 和网络直径 $D$, Kautz 图 $K(d, D)$ 是 可容纳节点数 $N\left(=d^{D}+d^{D-1}\right)$ 最多的拓扑图. 同 时, Kautz 图还具有最大连通度 (maximum connectivity)、常量拥塞和简单路由等优良特性.

显然, Kautz 图不能容纳任意数目的节点. 针 对该问题, 文献 [23] 基于同余运算 (congruent arithmetic) 方式提出了一般化 (generalized) 的 Kautz 图 定义. 令 $G K(d, n)$ 代表基为 $d$ 和节点数为 $n$ 的一 般化 Kautz 图, 那么其节点集 $V(G K(d, n))$ 和边集 $E(G K(d, n))$ 可以采用模 $n$ 的方式定义如下 ${ }^{[23]}$ :

$$
V(G K(d, n))=\{0,1, \ldots, n-1\},
$$$$
E(G K(d, n))=\{[i,(-d \times i-\alpha) \bmod n] \mid 0 \leqslant \alpha \leqslant d\} .
$$ 
需要注意的是, 基于同余运算的定义是原定义的 超集, 即对任意 $d$ 和 $D$, 我们有 $G K\left(d, d^{D}+d^{d-1}\right) \equiv$ $K(d, D)$.

给定两个具有 $n$ 和 $n^{\prime}$ 个节点的图 $G$ 和 $G^{\prime}$, 假 设 $n<n^{\prime}$. 从图 $G$ 到 $G^{\prime}$ 的可扩展度 (index of expandability) $\operatorname{ex}\left(G, G^{\prime}\right)$ 是指为得到图 $G^{\prime}$ 的一个子图, 从图 $G$ 中需要删除的最小边数 ${ }^{[22]} . \operatorname{ex}\left(G, G^{\prime}\right)$ 代表 了从 $G$ 得到 $G^{\prime}$ 的困难程度. 一般化 Kautz 图的可 扩展度为 $[23]$

$$
\operatorname{ex}(G K(d, n), G K(d, n+1))=\Theta(d n),
$$

显然该可扩展度对 $\mathrm{P} 2 \mathrm{P}$ 网络来说是不可行的.

线图 (line graphs) 的基本思想是所谓的边点变 换 ${ }^{[22]}$. 令图 $G$ 的点集和边集分别为 $V$ 和 $E$. 图 $G^{\prime}$ 被称为图 $G$ 的线图, 如果它的点集 $V\left(G^{\prime}\right)$ 和边 集 $E\left(G^{\prime}\right)$ 分别满足 $V\left(G^{\prime}\right)=\{u v \mid[u, v] \in E\}$, 并且 $E\left(G^{\prime}\right)=\left\{[u v, v w] \mid u v, v w \in V\left(G^{\prime}\right)\right\}$. 限于篇幅, 本文 不对其进行详细介绍. 对线图技术感兴趣的读者可 以参考文献 $[22,23]$.

\section{2 分布式 Kautz 图}

本节首先对分布式 Kautz 图的基本特点进行简 介, 然后提出分布式 Kautz 图的定义并对其进行分 析.

\section{1 概述}

静态拓扑图特性和动态拓扑维护机制是决定 P2P 网络性能的两个关键因素 ${ }^{[24]}$. Kautz 图具有 最优网络直径、常量度数以及常量拥塞等很多优良 特性. 但是由于在节点动态加入退出时拓扑维护的复 杂性, 现有的 $\mathrm{P} 2 \mathrm{P}$ 网络只能够基于以 2 为基 (base) 的 Kautz 图进行构建.

针对该问题, 本文提出一种分布式 Kautz 图. 直 观上, D-Kautz 图可以被看作是一种 “近似 Kautz 图”: 在一个基为 $d$ 的 D-Kautz 图中, 节点标识具 有相同的基 $d$, 但是其标识长度不同. 这是因为节点 动态加入退出的需要, 将在下一节介绍.

令 $x=x_{1} x_{2} \cdots x_{m}$ 是 D-Kautz 图 $G$ 中的节点. 那么节点 $x$ 的入边邻居具有如下形式:

$$
\alpha_{1} \cdots \alpha_{r} x_{1} \cdots x_{n-1} \in V(G),
$$

其中 $0 \leqslant r \leqslant 2, \alpha_{i} \in\{0,1,2, \ldots, d\}, \alpha_{i} \neq \alpha_{i+1}, \alpha_{r} \neq$ $x_{1}$; 节点 $x$ 的入边邻居具有如下形式:

$$
x_{t} \cdots x_{2} x_{3} \cdots x_{n} \beta \in V(G) \text {, }
$$

其中 $1 \leqslant t \leqslant 3, \beta \in\{0,1,2, \ldots, d\}, \beta \neq x_{m}$.

\section{2 定义}

下面首先简介一些基本概念, 然后提出 D-Kautz 图的定义.

定义 1 匹配度 (matching index). 对任意两 个 Kautz 串 $u=u_{1} u_{2} \cdots u_{m}$ 和 $v=v_{1} v_{2} \cdots v_{n}$, $u$ 和 $v$ 的匹配度 $M(u, v)=i$ 是指 $i$ 的最大取值 $(0 \leqslant i \leqslant \min (m, n))$, 使得对任意的 $j(1 \leqslant j \leqslant i)$ 有 $u_{m-i+j}=v_{j}$.

例如: $M(10121,012120)=4, M(10121,12120)=$ 3.

令 $u=u_{1} u_{2} \cdots u_{m}, v=v_{1} v_{2} \cdots v_{n}$, 并假设 $M(u, v)=i$. 定义 $u \oplus v=u_{1} u_{2} \cdots u_{m-i} v_{1} v_{2} \cdots v_{n}$, 例如: $10121 \oplus 12102=1012102$.

假设 $|u| \geqslant|v|$, 定义 $\operatorname{Trim}(u, j)=u_{j+1} \cdots$ $u_{m-1} u_{m}$, 其中 $j$ 为不大于 $m$ 的整数, 例如 $\operatorname{Trim}(10121,2)=121$. 显然如果 $|u|=|v|$, 那么 $\operatorname{Trim}(u,|u|-|v|)=u$.

令边点变换前的拓扑图为 $G$, 边点变换后的拓 扑图为 $G^{\prime}$. 我们首先介绍怎样把图 $G$ 中的一条边变 换为图 $G^{\prime}$ 中的点.

假设图 $G$ 中的一条边 $[u, v]$ 的两个端点（即 Kautz 串) $u$ 和 $v$ 满足 $|u| \geqslant|v|$, 并且 $[u, v]$ 被选中 将进行变换. 那么在图 $G^{\prime}$ 中对应边 $[u, v]$ 的节点 (以 $\mathbb{F}([u, v])$ 表示) 将被定义为

$$
\mathbb{F}([u, v])=\operatorname{Trim}(u,|u|-|v|) \oplus v .
$$

例如, 对于边 $[21,10]$, 通过变换后将得到 $\mathbb{F}$ ([21, 10]) $=\operatorname{Trim}(21,|21|-|10|) \oplus 10=210$; 对于 边 $[210,02]$, 通过变换后将得到 $\mathbb{F}([210,02])=$ $\operatorname{Trim}(210,|210|-|02|) \oplus 02=102$.

假设在图 $G$ 中节点 $v$ 的标识不比其任意直接 邻居节点的标识更长. 令节点 $v$ 在原图 $G$ 中的 出度和入度分别为 $d^{+}$和 $d^{-}$, 令节点 $v$ 在原图 $G$ 中的出边邻居集合和入边邻居集合分别为 $\left\{t^{j}\right\}, j=$ $0,1, \ldots, d^{+}-1$ 和 $\left\{u^{i}\right\}, i=0,1, \ldots, d^{-}-1$. 分布式 Kautz 图的定义如下:

定义 2 分布式 Kautz 图. 在变换 $G^{\prime}=\mathrm{D}$ - 
$\operatorname{Kautz}(G, v)$ 中, 删除节点 $v$ 以及所有与节点 $v$ 相连 的出边和入边, 并增加新的节点集 $\Delta(V)$ 和新的边集 $\Delta(E)$ 如下:

$$
\Delta(V)=\left\{\mathbb{F}\left(\left[u^{i}, v\right]\right)\right\},
$$

其中 $i=0,1, \ldots, d^{-}-1$,

$$
\Delta(E)=\left\{\left[u^{i}, \mathbb{F}\left(\left[u^{i}, v\right]\right)\right]\right\} \cup\left\{\left[\mathbb{F}\left(\left[u^{i}, v\right]\right), t^{j}\right]\right\},
$$

其中 $i=0,1, \ldots, d^{-}-1$, 并且 $j=0,1, \ldots, d^{+}-1$. 我们把该变换称为分布式 Kautz 图变换. 令图 $G_{0}$ 为 Kautz 图 $G_{0}=K(d, D)$, 我们把通过反复进 行 D-Kautz 变换得到的一系列拓扑图称为分布式 Kautz(D-Kautz) 图.

从定义 2 中容易得出, 在新的 D-Kautz 图 $G^{\prime}=$ $\operatorname{D-Kautz}(G, v)$ 中每一个新节点对应原图 $G$ 中节点 $v$ 的一条入边.

\section{3 分析}

下面我们将对 D-Kautz 图进行分析. 需要注意 的是, 限于篇幅, 在本文中仅对定理、引理等进行了 简明扼要地证明.

定理 1 令 D-Kautz 图 $G$ 的基为 $d$. 假设 $x=x_{1} x_{2} \cdots x_{m}$ 为图 $G$ 中的一个节点. 那么节点 $x$ 的入边邻居集合为

$$
\left\{s \mid s=\alpha_{1} \cdots \alpha_{r} x_{1} \cdots x_{n-1} \in V(G), 0 \leqslant r \leqslant 2\right\},
$$

其中 $a_{i} \in\{0,1,2, \ldots, d\}, \alpha_{i} \neq \alpha_{i+1}, \alpha_{r} \neq x_{1}$. 节点 $x$ 的出边邻居集合为

$$
\left\{t \mid t=x_{t} \cdots x_{2} x_{3} \cdots x_{n} \beta \in V(G), 1 \leqslant t \leqslant 3\right\},
$$

其中 $\beta \in\{0,1,2, \ldots, d\}, \beta \neq x_{m}$.

证明 定理 1 在初始状态 $G_{0}=K(d, D)$ 下成 立. 我们将证明如果定理 1 对图 $G_{i}$ 成立 $(i \geqslant 0)$, 那 么它将也对图 $G_{i+1}=\mathrm{D}-\operatorname{Kautz}\left(G_{i}, v\right)$ 成立.

令节点 $v$ 为 $v=v_{1} v_{2} \cdots v_{m}$. 根据 D-Kautz 图 的定义, 图 $G_{i}$ 中节点 $v$ 的任意入边邻居节点 $u$ 满足 $|u| \geqslant|v|$. 由于定理 1 对图 $G_{i}$ 成立, 图 $G_{i}$ 中节点 $v$ 的入边邻居节点具有如下形式:

$\alpha_{1} \cdots \alpha_{r} v_{1} v_{2} \cdots v_{m-1} \in V\left(G_{i}\right), \quad 0 \leqslant r \leqslant 1$.

因此, 根据 D-Kautz 变换的定义, 在图 $G_{i+1}$ 中 新增节点具有如下形式:

$\alpha v_{1} v_{2} \cdots v_{m} \in G_{i+1}, \alpha \in\{0,1,2, \ldots, d\}, \alpha \neq x_{1}$.

我们首先证明当节点 $x$ 是图 $G_{i+1}$ 中的一个新 节点 $x=\alpha v_{1} v_{2} \cdots v_{m}$ 时定理 1 对图 $G_{i+1}$ 成立.
对图 $G_{i+1}$ 中的新点 $v^{\prime}=\alpha v_{1} v_{2} \cdots v_{m}$, 令节 点 $y$ 为 $v^{\prime}$ 在图 $G_{i+1}$ 中的入边邻居, 根据 D-Kautz 图定义, 节点 $y$ 也为 $v$ 在图 $G_{i}$ 中的入边邻居, 从 而节点 $y$ 具有如下形式: $y=\alpha v_{1} v_{2} \cdots v_{m-1}$ 或 $y=\alpha^{\prime} \alpha v_{1} v_{2} \cdots v_{m-1}$.

令节点 $u=\alpha v_{1} v_{2} \cdots v_{m-1}$ 是图 $G_{i+1}$ 中的节点. 显然 $u$ 在图 $G_{i}$ 中是 $v$ 的入边邻居, 通过 D-Kautz 变 换, 将得到新点 $v^{\prime}=\alpha v_{1} v_{2} \cdots v_{m} \in V\left(G_{i+1}\right)$, 显然 $u$ 在图 $G_{i+1}$ 中是 $v^{\prime}$ 的入边邻居.

同理, 令节点 $\alpha^{\prime} \alpha v_{1} v_{2} \cdots v_{m-1}$ 是图 $G_{i+1}$ 中的 节点, 有 $u^{\prime}$ 在图 $G_{i+1}$ 中是 $\alpha v_{1} v_{2} \cdots v_{m} v^{\prime}$ 的入边邻 居.

如果图 $G_{i}$ 中存在 $u^{\prime \prime}=\alpha^{\prime \prime} \alpha^{\prime} \alpha v_{1} v_{2} \cdots v_{m}$, 那么 将不存在 $u^{\prime}=\alpha^{\prime} \alpha v_{1} v_{2} \cdots v_{m}$ 或 $u=\alpha v_{1} v_{2} \cdots v_{m-1}$, 因此, 图 $G_{i}$ 和图 $G_{i+1}$ 中均不存在形如 $u^{\prime \prime}=$ $\alpha^{\prime \prime} \alpha^{\prime} \alpha v_{1} v_{2} \cdots v_{m}$ 的点.

因此, 对图 $G_{i+1}$ 中任意新节点 $v^{\prime}=\alpha v_{1} v_{2}$ $\cdots v_{m} \in V\left(G_{i+1}\right)$, 所有形如 $\alpha_{1} \cdots \alpha_{r} x_{1} \cdots x_{m-1} \in$ $V\left(G_{i+1}\right)(0 \leqslant r \leqslant 2)$ 的节点都是节点 $v^{\prime}$ 的入边邻 居.

另一方面, 对图 $G_{i+1}$ 中新节点 $x=\alpha v_{1} v_{2}$ $\cdots v_{m} \in V\left(G_{i+1}\right)$ 的任意入边邻居 $y$, 根据定义 2 , $y$ 也是图 $G_{i}$ 中节点 $v$ 的入边邻居. 由于定理 1 对 图 $G_{i}$ 成立, 节点 $y$ 形如 $\alpha_{1} \cdots \alpha_{r} \alpha v_{1} v_{2} \cdots v_{m-1}$, 其 中 $0 \leqslant r \leqslant 1$. 因此新节点 $x$ 的入边邻居 $y$ 满足:

$y \in\left\{\alpha_{1} \cdots \alpha_{r} v_{1} \cdots v_{m-1} \in V\left(G_{i+1}\right), 0 \leqslant r \leqslant 2\right\}$.

图 $G_{i+1}$ 中新节点 $x=\alpha v_{1} v_{2} \cdots v_{m} \in V\left(G_{i+1}\right)$ 的入边邻居集合为

$$
\left\{s \mid s=\alpha_{1} \cdots \alpha_{r} x_{1} \cdots x_{n-1} \in V(G), 0 \leqslant r \leqslant 2\right\} .
$$

其中 $\alpha_{i} \in\{0,1,2, \ldots, d\}, \alpha_{i} \neq \alpha_{i+1}, \alpha_{r} \neq x_{1}$.

同理可证, 图 $G_{i+1}$ 中新节点 $x=\alpha v_{1} v_{2} \cdots v_{m} \in$ $V\left(G_{i+1}\right)$ 的出边邻居集合为

$$
\left\{t \mid t=x_{t} \cdots x_{2} x_{3} \cdots x_{n} \beta \in V(G), 1 \leqslant t \leqslant 3\right\} .
$$

其中 $\beta \in\{0,1,2, \ldots, d\}, \beta \neq x_{m}$. 因此, 当节点 $x$ 是 图 $G_{i+1}$ 中的新节点时, 定理 1 对图 $G_{i+1}$ 成立.

类似地, 当节点 $x$ 在图 $G_{i}$ 中是节点 $v$ 的出边邻 居或入边邻居时, 定理 1 对图 $G_{i+1}$ 成立.

与图 $G_{i}$ 比较, 在图 $G_{i+1}$ 中其他点的出边邻居 和入边邻居没有发生任何变化. 因此对图 $G_{i+1}$ 定理 
1 成立. 证毕.

基于 D-Kautz 图的定义以及定理 1 , 容易得 出如下结论: 在变换 $G^{\prime}=\operatorname{D-Kautz}(G, v)$ 后, 在 新图 $G^{\prime}$ 将增加 $d$ 个新节点 $\alpha v_{1} v_{2} \cdots v_{m}, \alpha \in$ $\{0,1,2, \cdots, d\}, \alpha \neq v_{1}$.

从定理 1 容易推出如下推论.

推论 1 在 D-Kautz 图中任给节点 $x=x_{1} x_{2}$ $\cdots x_{m}, x$ 至少有一个标识为 $\alpha_{1} \cdots \alpha_{r} x_{1} x_{2} \cdots x_{m-1}$ 的 入边邻居, 其中 $0 \leqslant r \leqslant 2$.

引理 1 令 D-Kautz 图 $G$ 的基为 $d$, 则图 $G$ 中 任意节点的出度为 $d$, 所有节点的平均入度为 $d$.

证明 显然对初始状态下的 $G_{0}=K(d, D)$, 上 述定理成立. 设在第 $i+1$ 次 D-Kautz 变换 $G_{i+1}=\mathrm{D}-$ $\operatorname{Kautz}\left(G_{i}, v\right)$ 前, 上述定理成立. 由定义 2 , 可知对图 $G_{i+1}$ 中的每个新点 $v^{\prime}, v^{\prime}$ 在图 $G_{i+1}$ 中的出度等于 $v$ 在图 $G_{i}$ 中的出度. 因此 $v^{\prime}$ 的出度为 $d$.

对图 $G_{i}$ 中点 $v$ 每个入边邻居 $u$, 令 $u$ 与 $v$ 在图 $G_{i+1}$ 中生成的新点为 $v^{\prime}$, 由 D-Kautz 图定义可知 $u$ 的出边邻居增加了 $v^{\prime}$ 并减少了 $v$, 因此 $u$ 的出度为 $d$.

与图 $G_{i}$ 比较, 在图 $G_{i+1}$ 中其他点的出边邻居 没有发生任何变化. 因此图 $G_{i+1}$ 中任意节点的出度 为 $d$.

由于所有节点的出度总和等于入度总和, 显然有 图 $G$ 中所有节点的平均入度为 $d$. 从而引理 1 成立.

引理 2 令 D-Kautz 图 $G$ 的基为 $d$ 、节点数为 $N$, 则图 $G$ 的网络直径 $D<2 \log _{d} N$.

证明 令 $D(u, v)$ 表示 D-Kautz 图 $G$ 中任意 两点 $u=u_{1} u_{2} \cdots u_{m}$ 和 $v=v_{1} v_{2} \cdots v_{n}$ 的距离. 设 $M(u, v)=i$, 令

$$
w=u \oplus v=u_{1} u_{2} \cdots u_{m} v_{i+1} v_{i+2} \cdots v_{n} .
$$

根据短路径路由策略 (将在下一节介绍), 路由 过程中的第 $j$ 个中间节点是 Kautz 串 $w$ 的一个子 串 $s=u_{k} u_{k+1} \cdots u_{m} v_{i+1} v_{i+2} \cdots v_{i+j}$. 注意到第 $j$ 个 中间节点的最右侧字符为 $v_{i+j}$, 因此在第 $n-i$ 步, 子串 $s$ 的最右侧字符为 $v_{n}$, 由于已知图 $G$ 中存在点 $v=v_{1} v_{2} \cdots v_{n}=u_{m-i+1} \cdots u_{m} v_{i+1} v_{i+2} \cdots v_{n}$, 因此 从 $u$ 到 $v$ 的距离满足 $D(u, v)=|v|-M(u, v) \leqslant|v|$.

令节点 $y$ 为图 $G$ 中标识长度最长的节点. 显然 图 $G$ 的直径满足 $D \leqslant n$.
另一方面, 在静态 Kautz 图中有 $N=d^{D}+d^{D-1}$. 令图 $G$ 中标识长度最短的节点为 $x$, 由于每次变换 $G_{i+1}=\mathrm{D}-\operatorname{Kautz}\left(G_{i}, w\right)$ 将在图 $G_{i+1}$ 中使用 $d$ 个标 识长度为 $|w|+1$ 的新节点代替图 $G_{i}$ 中的节点 $w$, 因 此有 $N>d^{|x|}$, 即 $|x|<\log _{d} N$. 进而有图 $G$ 中节点 $y$ 到节点 $x$ 的距离满足 $D(y, x)=|x|-M(y, x) \leqslant|x|$.

由定理 1, 易知图 $G$ 中相邻两个节点的标识长 度之差不大于 1 , 显然有 $y$ 与 $x$ 标识长度之差不大于 $y$ 与 $x$ 之间的距离, 即 $|y|-|x| \leqslant D(y, x)$, 进而图 $G$ 的网络直径满足 $D<2 \log _{d} N$. 从而引理 2 成立.

定理 2 令图 $G$ 为基为 $d$, 节点数为 $N$ 的 DKautz 变换, 则图 $G$ 的网络直径 $D<2 \log _{d} N$. 图 $G$ 中任意节点的出度为 $d$, 所有节点的平均入度为 $d$.

证明 该理可以直接从引理 1 和 2 推出.

\section{$3 \mathrm{SKY}$ 系统设计}

下面我们将应用 D-Kautz 图技术, 提出一种能 够基于以任意常数为基的 Kautz 图进行构建的有效 的 P2P 网络—SKY. SKY 需要处理如下 P2P 相 关操作: 资源命名与查找、消息路由、以及处理节点 动态加入退出的维护机制.

\section{1 资源命名、发布与查找}

SKY 中各资源对象的标识为一个基为 $d$ 长度 为 $m$ 的 Kautz 串. 我们在以前的工作 ${ }^{[20]}$ 中提 出了能够生成基为 2 的目标 Kautz 串的 Hash 算 法 Kautz_hash, 并证明了所生成的目标 Kautz 串在 Kautz 空间中均匀分布. 该算法能够容易地扩展为 支持任意基 Kautz 串的生成, 具体如图 2 所示.

下文中我们将使用 “节点” 表示 D-Kautz 图中的 节点及节点标识, 可以从上下文区分其含义. SKY 中 资源的发布基于如下匹配策略 (matching index policy).

匹配策略 给定目标 Kautz 串 $s=s_{1} s_{2} \cdots s_{r}$, 在 SKY 系统中该 Kautz 串有唯一负责节点 $u=$ $u_{1} u_{2} \cdots u_{m}$ 满足

$$
\begin{aligned}
& \forall t \in G, M(t, s) \leqslant M(u, s), \\
& M(u, s)=|u|, \text { 或 } M(u, s)=|u|-1 \wedge \Delta(u, s)=0 . \\
& \Delta \text { 的定义为 } \Delta(u, s)=\mid\left(u_{m-i} \Theta u_{m-i+1}\right)- \\
& \left(s_{i+1} \Theta s_{i}\right) \mid \text {. 其中运算 } \Theta \text { 定义为若 } x<y, \text { 则 } x \Theta y=x ; \\
& \text { 否则 } x \Theta y=x-1 .
\end{aligned}
$$


Procedure Kautz_hash_E (Key $\boldsymbol{X}$, Base $\boldsymbol{d}$, Length $\boldsymbol{m}$ )

/ ***** 资源命名算法 $* * * * * /$

1 str $\leftarrow$ Base_hash $(X, d)$;

2 while $(|\operatorname{str}|<m)\{$

// 如果 $\operatorname{str}$ 的长度小于要求

3 str $\longleftarrow$ str + Base_hash $($ str, $d) ;\}$

// 反复调用 hash 函数

4 str $\leftarrow$ Get_Left_String $($ str, $m)$;

$/ /$ 取前 $m$ 个字符

5 return str;

Procedure Base_hash(Key $\boldsymbol{k}$, Base $\boldsymbol{b}$ )

/***** 基于 $k$ 产生基为 $b$ 的 Kautz 字符串 $* * * * *$ /

$1 s \leftarrow$ SHA-1 $(k)$;

// 基于 SHA-1 得到二进制字符串

$2 s \leftarrow$ Binary_to_b_plus_1 $(s, b)$;

$/ /$ 转换为 $(b+1)$ 进制

$3 s \leftarrow$ Combine_same_neighbor_bits $(s)$;

// 合并相同的相邻字符

4 return $s$;

// 返回结果

图 2 SKY 资源命名算法

在 $\Delta$ 的定义中, 若 $u$ 和 $v$ 的匹配度为 $i$, 即

$$
u_{m-i+1} u_{m-i+2} \cdots u_{m}=v_{1} v_{2} \cdots v_{i} \text {, }
$$

那么 $u_{m-i} \Theta u_{m-i+1}$ 代表了: 在所有后缀为 $u_{m-i+1}$ $u_{m-i+2} \cdots u_{m}$ 的节点中, 第 $m-i$ 位是 $u_{m-i}$ 的节 点的相对位置; 同理, $v_{i+1} \Theta v_{i}$ 代表了: 在所有前缀为 $v_{1} v_{2} \cdots v_{i}$ 的 Kautz 串中, 第 $i+1$ 位是 $v_{i+1}$ 的 Kautz 串的相对位置. 进而, $\Delta(u, v)$ 代表了节点标识 $u$ 和资 源对象标识 $v$ 的相对位置的差异.

需要注意的是, 简单的前缀或后缀匹配策略 (prefix/suffix matching policy) ${ }^{[13,20,21]}$ 不适用于 SKY. 详见附录 A 中的分析.

如果节点 $p$ 需要发布资源 $O$, 那么它将首先得 到资源 $O$ 的目标 Kautz 串 $s$. 进而节点 $p$ 将发起一 次到目标 Kautz 串 $s$ 的路由 (路由算法将在下一节 介绍), 该路由将最终到达符合上述匹配策略的唯一 节点 $u$. 资源 $O$ 将被发布到节点 $u$.

例如, 令图 $G$ 为如图 1(b) 所示的 Kautz 图 $K(2,2)$. 在 $G^{\prime}=\mathrm{D}-\operatorname{Kautz}(G, 10)$ 中, 令节点 $u=010$, 节点 $u^{\prime}=210$, 设目标 Kautz 串 $t=010201, s=$ 101201, $s^{\prime}=102101$.

1) 由于 $M(u, t)=3=|u|$, 因此以 $t$ 为目标 Kautz 串的路由消息最终将到达节点 $u$;

2) 由于 $\Delta(u, s)=0, \Delta\left(u^{\prime}, s\right)=1$, 因此虽然节点 $u$ 和 $u^{\prime}$ 都与 $s$ 具有最大匹配度 $M(u, s)=M\left(u^{\prime}, s\right)=$ 2 , 以 $s$ 为目标 Kautz 串的路由消息最终将到达节点 $u$;

3) 同理, $M\left(u, s^{\prime}\right)=M\left(u^{\prime}, s^{\prime}\right)=2, \Delta\left(u, s^{\prime}\right)=1$, $\Delta\left(u^{\prime}, s^{\prime}\right)=0$, 以 $s^{\prime}$ 为目标 Kautz 串的路由消息最终 将到达节点 $u^{\prime}$.

资源查找过程与发布过程类似, 这里不再赘述.

\section{2 路由算法}

SKY 采用了如下短路径路由策略 ${ }^{[20]}$ : 设目标 Kautz 串为 $s$, 路由消息当前路由至中间节点 $u^{\prime}$, 那 么其下一跳节点 $u^{\prime \prime}$ 应满足 $M\left(u^{\prime \prime}, s\right)=M\left(u^{\prime}, s\right)+1$. 路由消息最终将到达与目标 Kautz 串具有最大匹配 度的节点.

然而, 根据上一节的分析, 系统中可能有多个 节点与 Kautz 串 $s$ 具有相同最大匹配度. 在这种 情况下, 路由消息将被转发一个邻居节点 $w$, 使得 $M(w, s)=M(u, s)$ 并且 $\Delta(w, s)=0$. 具体过程如图 3 所示.

按照图 3 所示的路由算法, 以 Kautz 串 $v=$ $v_{1} v_{2} \cdots v_{n}$ 为目标的路由消息最终会到达节点 $u$. 直 观上, 上述路由算法只能保证 $u$ 与 $v$ 具有局部最大 的匹配度, 然而, 定理 3 保证了 SKY 路由算法的正 确性 (详见 3.4 小节).

\section{3 维护机制}

本节首先对 D-Kautz 变换进行改进, 以支持任 意节点数的 $\mathrm{P} 2 \mathrm{P}$ 拓扑图, 然后介绍 $\mathrm{SKY}$ 中处理节点 加入退出的维护机制. 
Procedure Message_routing (CurrentNode $\boldsymbol{u}$, DestString $\boldsymbol{s}$ )

$/ * * * * * * * * * *$ 节点 $u$ 转发到目标 Kautz 串 $s$ 的路由消息 $* * * * * * * * * * /$

1 if $(\exists t \in u$.OutNeighbors 使得 $M(t, s)>M(u, s))\{$

2 return Message_routing $(t, s) ;\}$

// 转发给匹配度更大的邻居

3 else if $\left(\exists t^{\prime} \in u\right.$.SibNeighbors

$$
\text { 使得 } \left.\left(M\left(t^{\prime}, s\right)=M(u, s)\right) \wedge\left(\Delta\left(t^{\prime}, s\right)=0\right)\right)\{
$$

4 return Message_routing $\left.\left(t^{\prime}, s\right) ;\right\}$

$/ /$ 转发给匹配度相同但 $\Delta$ 值为 0 的邻居

5 else \{

$6 \quad$ return $u ;\}$

// 当前节点即为目标节点

\section{图 3 SKY 路由算法}

\subsection{1 支持任意节点数的拓扑图}

根据 D-Kautz 图的定义和定理 1, 容易得出 每次变换 $G_{i+1}=\mathrm{D}-\operatorname{Kautz}\left(G_{i}, v\right)$ 后，新图 $G_{i+1}$ 中增加了 $d$ 个新节点 $\alpha v_{1} v_{2} \cdots v_{m} \in G_{i+1}, \alpha \in$ $\{0,1,2, \ldots, d\}, \alpha \neq x_{1}$. 因此, 当基 $d>2$ 时, D-Kautz 变换无法直接应用于 $\mathrm{P} 2 \mathrm{P}$ 系统的动态维护.

通过对 D-Kautz 变换进行简单的修改即可解决 该问题. 在每次变换后 SKY 把 $d$ 个新节点分成两份. 前半部分节点将合并成一个节点 $s$, 后半部分节点将 合并成一个节点 $t$. 合并成节点 $s$ (或 $t)$ 的节点称为 $s$ (或 $t$ ) 的子节点 (sub-node), 而节点 $s$ (或 $t$ ) 的子节 点数则称为 $s$ (或 $t$ ) 的秩 (order). 与之类似, 如果节 点 $v$ 的子节点数大于 1 , 那么节点 $v$ 将把它的子节点 分离成两份, 而不再进行 D-Kautz 变换. 节点 $v$ 负责
前半部分子节点; 新节点则负责剩余子节点.

通过上述方法, SKY 能够支持任意数目的节点. 显然, 每一个 SKY 拓扑有一个对应 (corresponding) 的 D-Kautz 图.

\subsection{2 节点加人}

当一个新节点 $p$ 加入 SKY 时，节点 $p$ 首先 需要查找一个负责此次加入的负责点. $p$ 首先通 过资源命径算法得到一个基为 $d$ 的目标 Kautz 串 $s=s_{1} s_{2} \cdots s_{r}$, 然后从某加入点出发, 路由至负责 $s$ 的节点 $u$. 然后节点 $u$ 发出 JOIN 消息, 在中间 节点 $u^{\prime}$, JOIN 消息将被转发给满足 $|w|<\left|u^{\prime}\right|$ 或 $\left(|w|=\left|u^{\prime}\right|\right) \wedge\left(w\right.$.order $>u^{\prime}$.order $)$ 的邻居节点 $u^{\prime}$.

上述过程如图 4 所示.

然后 SKY 将通过前面介绍的拓扑维护操作, 更

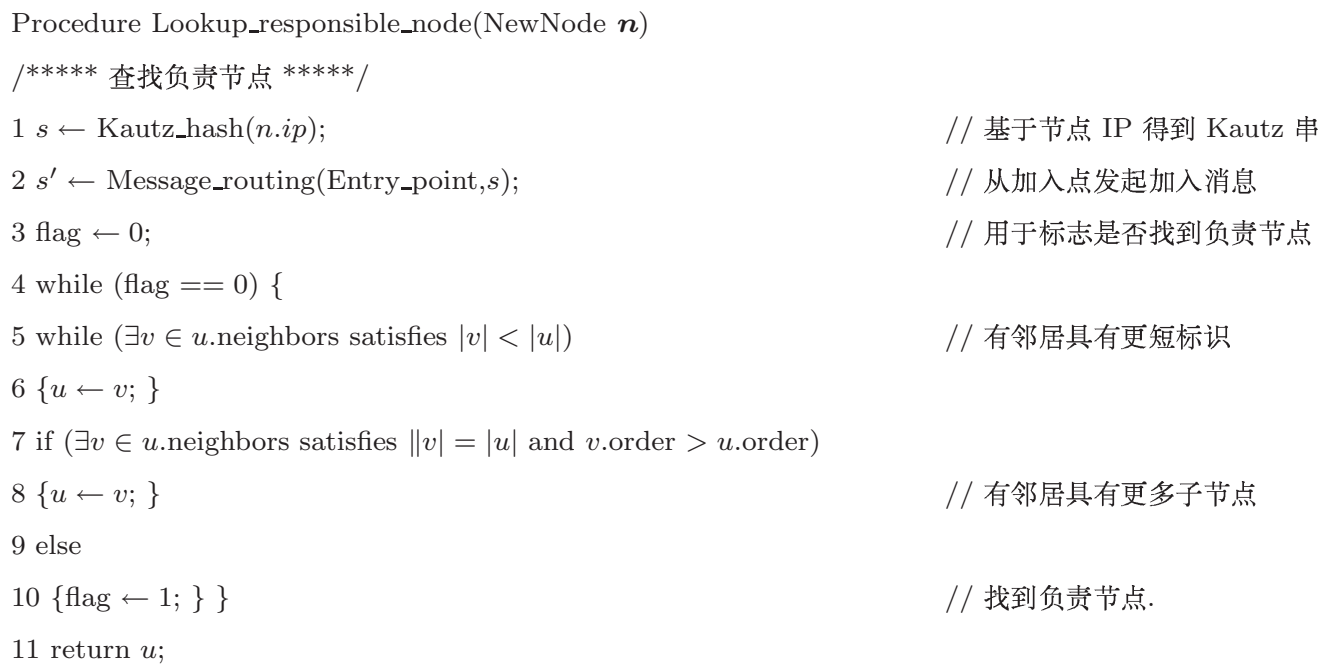


新相关节点的路由表, 其中负责节点 $v$ 即为通过 JOIN 消息的终点. 经过这一步拓扑图中将增加两 个新节点, 其中第 1 个节点对应负责节点 $v$, 第 2 个 节点对应新加入系统的节点 $p$.

该过程包含在图 5 和 6 中.

然后 SKY 将对资源进行重新分配. 显然与分离 操作或合并操作相关的资源分配可以随着子节点的 分配而完成, 因此下面我们只需介绍由变换 $G^{\prime}=\mathrm{D}-$ $\operatorname{Kautz}(G, v)$ 引起的资源分配. 该过程也包含在图 5 和图 6 中.

令 $v=v_{1} v_{2} \cdots v_{m}$, 在图 $G^{\prime}$ 中将有 $d$ 个新子节 点 $x=\alpha v_{1} v_{2} \cdots v_{m}, \alpha \in\{0,1,2, \ldots, d\}, \alpha \neq v_{1}$. 假设 新节点 $x$ 由由边 $[t, v]$ 生成, 那么根据资源对象与节 点的映射规则, $x$ 将获得如下两类资源对象.
1) 对 $t$ 的每个资源对象 $O$ (令其标识为 $R$ ), 如果 有 $M(x, R)>M(t, R)$, 那么 $O$ 将从 $t$ 转移至 $x$. 例 如, 在 Kautz 图 $G=K(2,2)$ 中节点 21 所负责的所 有形如 $210 *$ 的资源, 在变换 $G^{\prime}=\operatorname{D-Kautz}(G, 10)$ 后, 将转移至图 $G^{\prime}$ 中的新节点 210 .

2) 显然, 对 $v$ 的任意资源对象 $O$ (令其标识为 $R)$, 有 $M(v, R)=M(x, R)$. 由于 $v$ 将被新生成的 $d$ 个节点所代替, 因此如果 $\Delta(x, R)=0$, 那么 $R$ 将从 $v$ 转移至 $x$. 例如, 在 Kautz 图 $G=K(2,2)$ 中节点 10 所负责的所有形如 $102 *$ 的资源, 在变换 $G^{\prime}=\mathrm{D}-$ $\operatorname{Kautz}(G, 10)$ 后, 将转移至图 $G^{\prime}$ 中的新节点 210 (由 于 $\Delta(210,102 *)=0)$, 而图 $G$ 中节点 10 所负责的所 有形如 101* 的资源, 将转移至图 $G^{\prime}$ 中的新节点 010 (由于 $\Delta(010,101 *)=0)$.

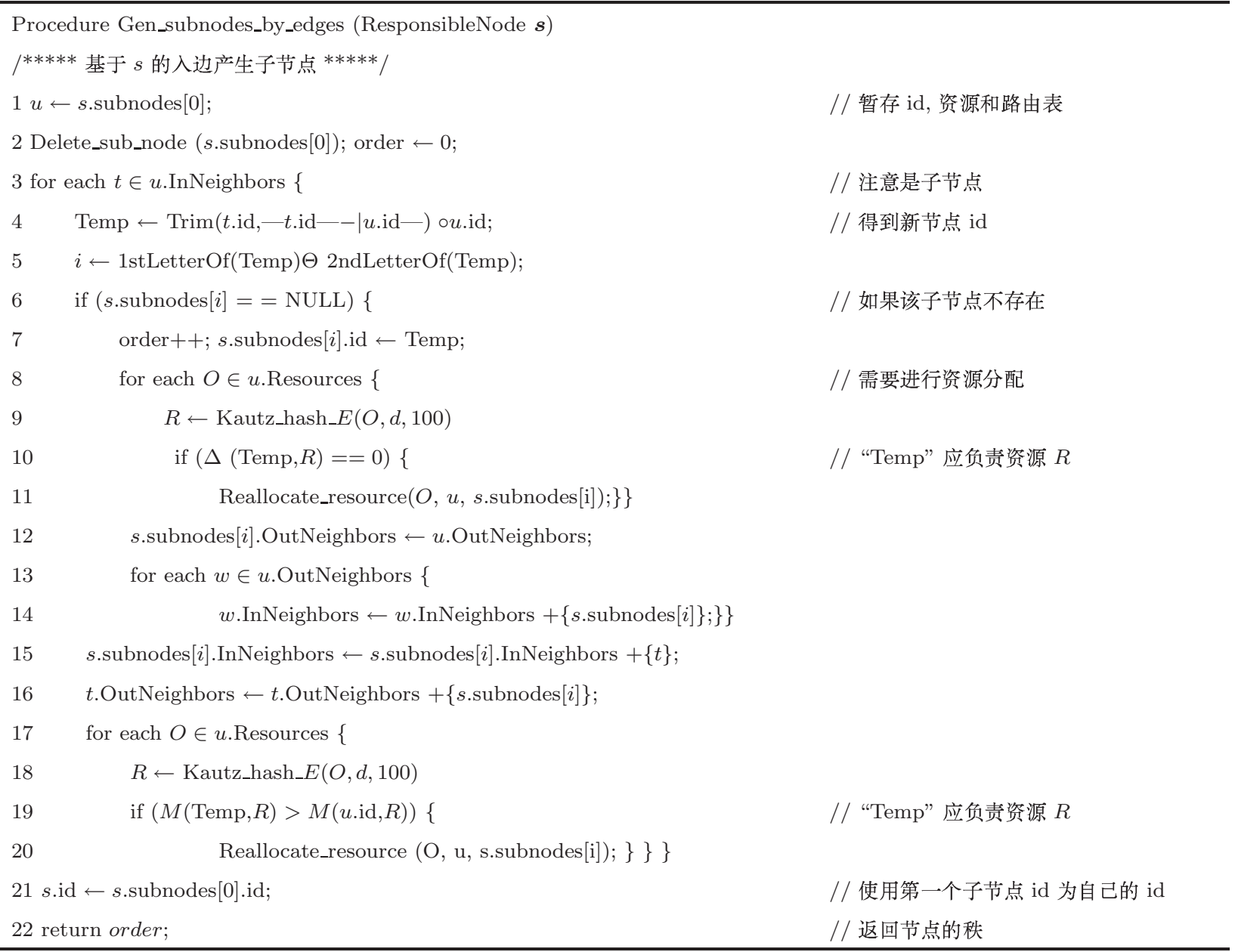

\section{图 5 产生子节点算法}




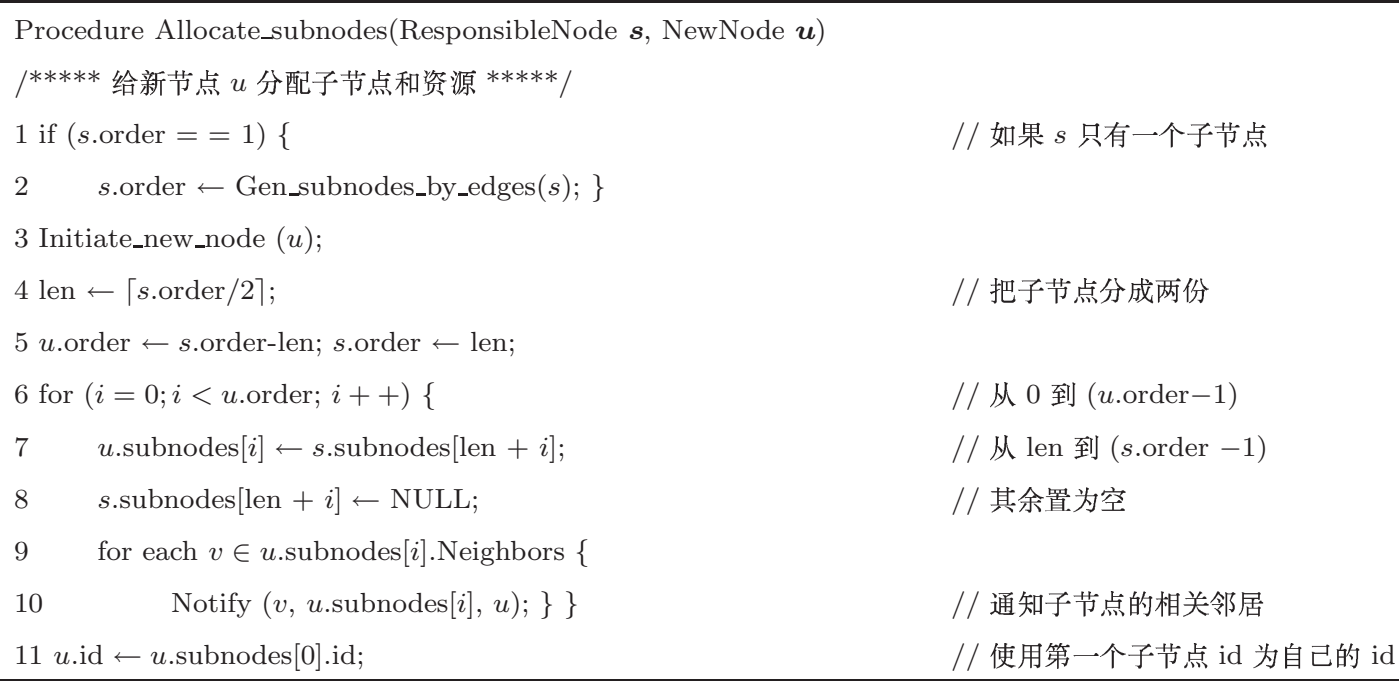

\section{图 6 子节点分配算法}

当多个节点同时加入或退出引起路由表更新时, SKY 的节点加入 (退出) 处理算法可能会导致部分 节点的路由表出现暂时的不一致情况. 为避免这种 情况, 当节点加入 (退出) 引起一次路由表更新时, 一个节点只处理一个节点加入 (退出) 消息, 只有所 有相关节点都完成了此次加入 (退出) 消息的处理 后, 再处理其他的加入 (退出) 消息. 上述过程与 FissionE ${ }^{[20]}$ 对节点并发加入退出的处理过程类似, 不 再赘述.

\subsection{3 节点退出}

当节点 $p$ 退出系统时, 其路由信息和资源都将 由系统中的其他节点负责. 该过程可以看作节点加 入的逆过程. 在 SKY 系统中每个节点向它的所有邻 居周期性地发送 Alive 消息. 通常情况下, 节点退出 前应主动发出通告消息, 把自己负责的资源对象、路 由信息等交给其他节点负责. 如果节点没有进行上 述处理就直接离开系统, 该节点的邻居节点将能够 很快发现该节点已退出, 并代替它进行相关处理.

节点退出的处理过程如图 7 所示.

\section{4 分析}

引理 3 在 SKY 中, 假设以 $x=x_{1} x_{2} \cdots x_{n}$ 为 目标 Kautz 串的消息最终路由至节点 $u$, 则节点 $u$ 满 足 $M(u, x)>|u|-2$.

证明 令 $u=u_{1} u_{2} u_{3} \cdots u_{m}$, 根据定理 1 , 容易 推出当 $M(u, x) \leqslant m-2$ 时, 节点 $u$ 总有一个出边邻
居 $v$, 使得 $M(v, x)=M(u, x)+1$, 因此若路由消息 终止于节点 $u$, 则必有 $M(u, x)>m-2$. 从而引理 3 成立.

定理 3 (SKY 路由正确性) 在 SKY 中, 从任 意节点发出的以 $x$ 为目标 Kautz 串的消息, 最终将 路由至系统中的唯一节点 $u$, 节点 $u$ 满足 SKY 的匹 配策略.

证明 令 $u=u_{1} u_{2} u_{3} \cdots u_{m}$. 由引理 3 , 可知 $M(u, x)=m-1$, 或 $M(u, x)=m$.

首先讨论 $M(u, x)=m-1$ 的情况. 由于路 由消息终止在 $u$, 由定理 1 , 易知 Kautz 串 $x$ 满 足 $x=u_{2} u_{3} \cdots u_{m} x_{m} \cdots x_{n}$. 显然节点 $u$ 满足 $\Delta(u, x)=0$. 下面我们只需证明系统中没有节点 $w$ 满足 $M(w, x) \geqslant m$.

否则系统中一定存在节点 $w=s u_{2} u_{3} \cdots u_{m} x_{m}$ $x_{m+1} \cdots x_{m+i}$, 其中 $s$ 是一个可为空的字符串, $i \geqslant$ 0. 显然 $w$ 有一个入边邻居 $w^{\prime}=s^{\prime} u_{2} u_{3} \cdots u_{m}$ $x_{m} x_{m+1} \cdots x_{m+i-1}$, 其中 $s^{\prime}$ 是可为空的字符串. 以 此类推, 我们可得到节点 $u^{\prime}=s^{\prime \prime} u_{2} u_{3} \cdots u_{m}$.

若 $s^{\prime \prime}$ 是空串, 则 $u^{\prime}=u_{2} u_{3} \cdots u_{m}$, 显然矛盾. 否 则若 $s^{\prime \prime}$ 不是空串, 给定 $x_{m}$, 节点 $u$ 只有一个出边 邻居 $v=u_{t} \cdots u_{2} u_{3} \cdots u_{m} x_{m}, 1 \leqslant t \leqslant 3$, 由于消息 路由终止于节点 $u, v$ 只能为 $v=u_{3} \cdots u_{m} x_{m}$. 由于 $|v|<|u|$, 给定 $u_{2}$, 则:

1）节点 $v$ 有一个入边邻居 $v^{\prime}=u_{2} u_{3} \cdots u_{m}, v^{\prime}$ 


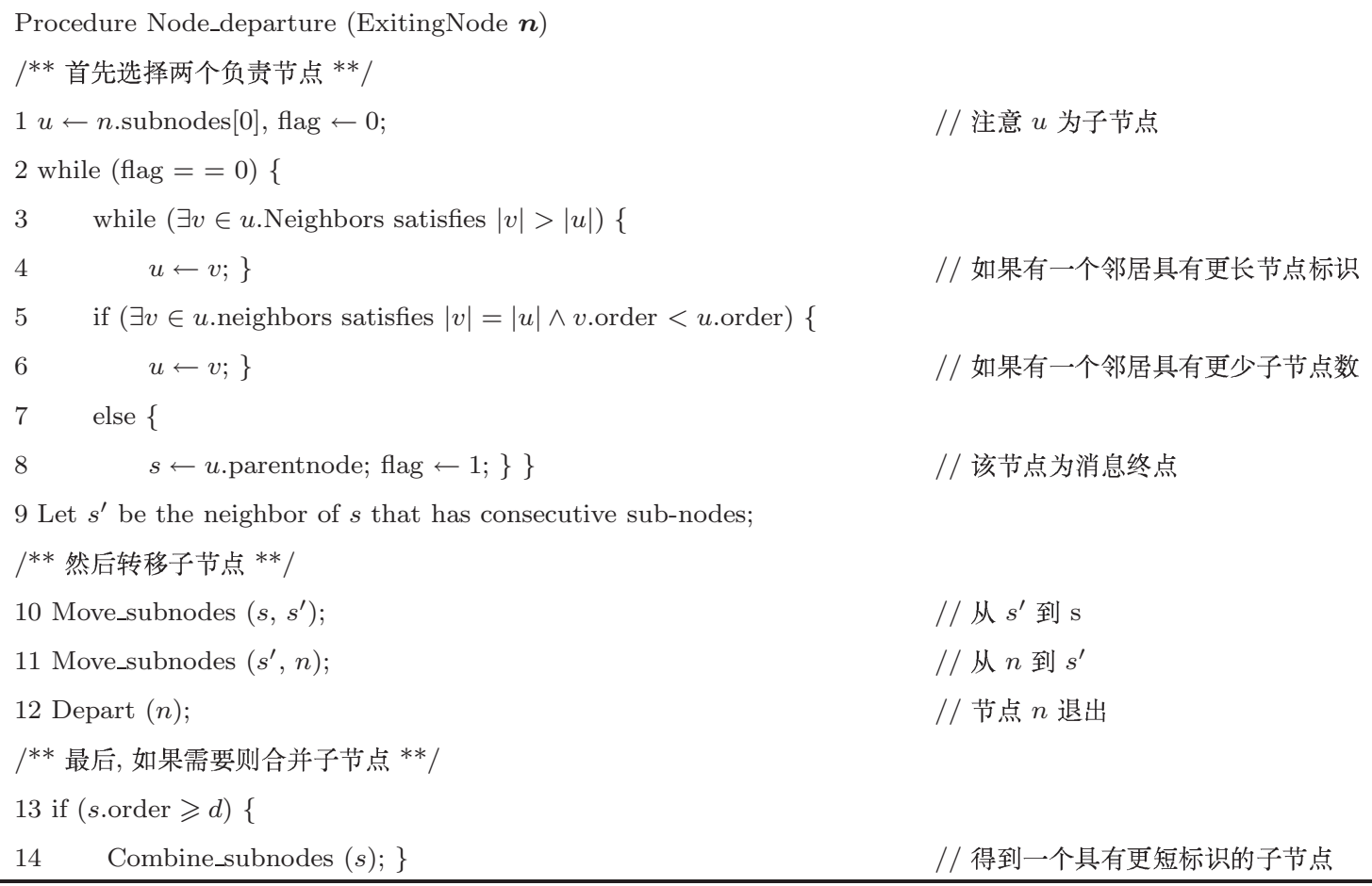

\section{图 7 节点退出处理算法}

是 $u$ 的子串, 矛盾;

2) 或 $v$ 有 $d$ 个入边邻居 $v^{\prime}=\alpha u_{2} u_{3} \cdots u_{m}, \alpha=$ $0,1, \ldots, d, \alpha \neq u_{2}$ :

如果 $\left|v^{\prime}\right|>\left|u^{\prime}\right|$, 则 $s^{\prime \prime}$ 为空且 $u^{\prime}=u_{2} u_{3} \cdots u_{m}$, $u^{\prime}$ 是 $u$ 的子串, 矛盾.

如果 $\left|v^{\prime}\right|<\left|u^{\prime}\right|$, 则 $d$ 个新节点中的一个一定是 $u^{\prime}$ 的字串, 矛盾.

如果 $\left|v^{\prime}\right|=\left|u^{\prime}\right|$, 则 $u^{\prime}$ 是 $d$ 个新节点之一且节点 $v$ 是 $u^{\prime}$ 的一个出边邻居. 然而, 节点 $u^{\prime}$ 已经有一个 出边邻居 $s^{\prime \prime \prime} u_{2} u_{3} \cdots u_{m} x_{m}$, 矛盾.

因此当 $M(u, x)=m-1$ 时定理成立.

同理可证当 $M(u, x)=m$ 时定理成立. 从而定 理 3 成立.

引理 4 令 SKY 拓扑图的基为 $d$, 那么 SKY 系 统中任意节点的出度为 $d$, 并且所有节点的平均入度 为 $d$.

证明 给定 SKY 中的任意节点 $x$, 显然 $x$ 的所 有子节点在 D-Kautz 图中具有相同的出边邻居. 进 而节点 $x$ 的出度与它任意子节点的出度相同, 所以 $x$ 的出度为 $d$.
由于所有节点的入度总和与出度总和相同, 因此 系统中所有节点的平均入度为 $d$. 从而引理 4 得证.

引理 5 令 SKY 拓扑图的基为 $d$, 节点数为 $N$. 那么 SKY 的网络直径为 $O\left(\log _{d} N\right)$.

证明 令 $x$ 为目标 Kautz 串. 假设消息产生在 节点 $v$, 并且路由终点为节点 $u$. 根据匹配策略, 我们 有 $M(u, x)=|u|$, 或 $M(u, x)=|u|-1$ 且 $\Delta(u, x)=0$.

如果 $M(u, x)=|u|$, 显然路由过程中的任意中 间节点 $u^{\prime}$ 都有一个邻居节点 $v^{\prime}$ 满足 $M\left(v^{\prime}, x\right)=$ $M\left(u^{\prime}, x\right)+1$. 从而路径长度满足:

$$
M(u, x)-M(v, x) \leqslant M(u, x)=|u| .
$$

如果 $M(u, x)=|u|-1$ 并且 $\Delta(u, x)=0$, 我们 需要考虑如下两种情况.

如果在最后一步之前中间节点 $u^{\prime}$ 满足 $M\left(u^{\prime}\right.$, $x)=|u|-1$ 且 $\Delta\left(u^{\prime}, x\right) \neq 0$, 那么路径长度满足:

$$
M\left(u^{\prime}, x\right)-M(v, x)+1 \leqslant M\left(u^{\prime}, x\right)+1=|u| .
$$

否则在最后一步之前中间节点 $u^{\prime}$ 满足 $M\left(u^{\prime}, x\right)=$ $|u|-2$, 那么路径长度满足:

$$
\begin{gathered}
M\left(u^{\prime}, x\right)-M(v, x)+1 \leqslant M\left(u^{\prime}, x\right)+1=|u|-1 . \\
\text { 令 } G^{*} \text { 为图 } G \text { 所对应的 D-Kautz 图. 对任意 }
\end{gathered}
$$


$x \in V(G)$ 我们有 $x$.order $\leqslant d / 2$. 因此 $G *$ 的节点数 满足 $N^{\prime}<d \times N$.

由定理 $2, \mathrm{SKY}$ 的网络直径满足 $D \leqslant|u|=$ $O\left(\log _{d} N\right)$. 从而引理 5 得证.

定理 4 (SKY 拓扑特性) 令 SKY 拓扑图的 基为 $d$, 节点数为 $N$. 那么 SKY 的网络直径为 $O\left(\log _{d} N\right)$. 系统中任意节点的出度为 $d$, 并且所有 节点的平均入度为 $d$.

证明 该定理可以直接从引理 4 和 5 推出.

从上述定理容易推出, SKY 系统中所有节点的 平均度数为 $2 d$.

定理 5 (SKY 维护开销) SKY 系统中节点加 入退出的消息开销为 $O\left(\log _{d} N\right)$.

证明 根据节点加入的处理过程, 当新节点 $p$ 加入系统时, 首先需要路由至目标 Kautz 串 $s$. 显然 该消息的传播距离小于系统网络直径.

然后系统将转发一个 JOIN 消息, 直到到达节点 $v$, 使得没有一个邻居的标识比 $v$ 更短, 而且在具有 相同标识长度的邻居中没有一个邻居的逻辑节点数 比 $v$ 更多. 显然, 对任意两个节点 $u$ 和 $v$, 我们有

$$
\begin{aligned}
& || v|-| u|| \leqslant|u|<\log _{d} N \wedge \\
& \mid v \text {.order }-u \text {.order } \mid \leqslant\lceil d / 2\rceil \leqslant d-1 .
\end{aligned}
$$

此外, 根据定理 4 , 系统中每个节点平均有 $2 d$ 个 邻居节点, 从而最多需要通知 $2 d$ 个节点此次拓扑变 化. 因此, SKY 系统中节点加入所引起的消息开销为 $O\left(\log _{d} N\right)$.

同理, SKY 系统中节点退出所引起的消息开销 同样为 $O\left(\log _{d} N\right)$. 从而定理 5 得证.

\section{4 模拟验证}

我们在 FissionE 模拟器 ${ }^{[20]}$ 中实现了 SKY, 并 对 SKY 的节点度数分布、网络直径和平均路径长度, 以及维护开销等进行了模拟分析.

\section{1 节点度数}

根据定理 4, SKY 中任意节点的出度为常数 $d$. 因此在本节中我们仅对系统中节点入度的分布进行 了模拟分析. 模拟中 SKY 的基 (base) 设置为 3, 节 点个数分别为 $4 \mathrm{~K} / 32 \mathrm{~K}$. 模拟结果如图 8 所示.

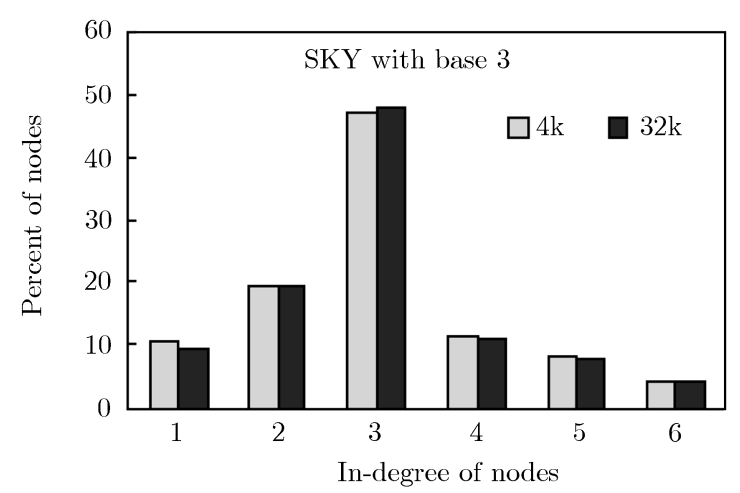

图 $8 \mathrm{SKY}$ 中的节点人度分布 $(\mathrm{base}=3)$

从图 8 中我们可以看出, SKY 中的节点入度分 布在 1 至 6 之间, 大多数节点的入度接近 SKY 的基 $d(=3)$.

\section{2 网络直径和平均路径长度}

本节将对 SKY 的网络直径和平均路径长度进行 模拟分析. 在每一次实验中, 我们首先随机选择一个 开始节点和一个目标 Kautz 串, 然后从开始节点发 送一个以该 Kautz 串为目标的消息, 并采用图 3 所 示的路由算法进行路由.

在实验中我们设置 SKY 系统中的节点个数从 256 到 $32 \mathrm{~K}$, 而 SKY 系统的基则设置为 3. 我们 把 SKY 与其他常量度数的 P2P 网络 (CAN 和 FissionE) 进行了比较. 模拟结果是 10000 次实验的平 均值. 如图 9 所示.

在图 9 中 SKY 的网络直径和平均路径长度分别 表示为 SKY (max) 和 SKY (avr). 从图中可以看出, SKY 的平均路径长度非常接近 $\log _{d} N$, 而网络直径 则略高于 $\log _{d} N$. 当节点个数超过 512 时, SKY 的平 均路径长度小于 CAN. FissionE 仅支持以 2 为基的 Kautz 图. 与 FissionE 比较, 在系统具有更高网络带 宽的时候, SKY 能够采用更高的 $d$ 作为初始 Kautz 图的基, 从而具有更好的性能.

\section{3 维护开销}

本节将对 SKY 系统中节点加入退出时的动态 维护开销进行模拟分析. SKY 的基设为 $d=3$, 节点 个数设为 $N=32 \mathrm{~K}$. 在每次实验中, 一个新节点首先 通过一个随机选择的加入点来加入系统, 然后随机选 择一个已经存在的节点退出系统.

图 10 展示了 SKY 动态维护机制的平均消息开 
销 (包括 JOIN/DEPART 消息转发的步数以及通知 邻居节点所需消息数). 在图 10 中同时展示了相同 设置下 $\mathrm{CAN}$ 的动态维护开销. 显然, 与 $\mathrm{CAN}$ 比较, SKY 具有更低的动态维护开销.

文献 [20] 已经证明, Kautz 图的拥塞特性为 $(1+o(1))$-congestion-free, 因此限于篇幅, 本文省略了 对 SKY 系统拥塞特性的模拟验证.

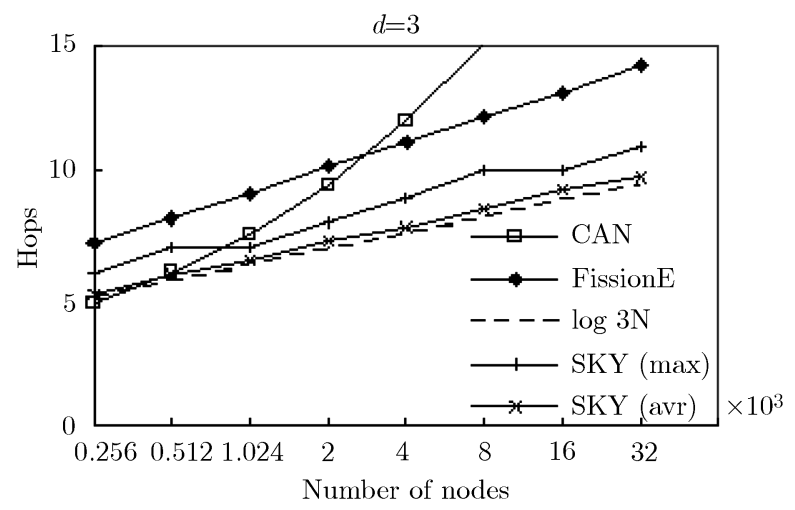

图 $9 \mathrm{SKY}$ 的网络直径和平均路径长度

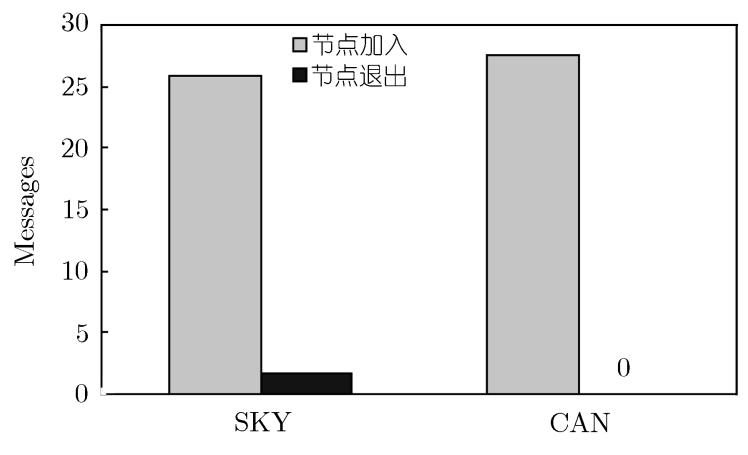

图 $10 \mathrm{SKY}$ 的维护开销

\section{5 相关工作}

\subsection{FissionE}

我们在以前的工作 ${ }^{[20]}$ 中提出了 FissionE. FissionE 使用 Kautz 图 $K(2, D)$ 作为静态拓扑, 每个节 点的标识都是一个基为 2 的 Kautz 串. FissionE 采 用区域拆分与合并方式来处理节点的动态加入退出 情况. 对 FissionE 中的任一节点 $u=u_{1} u_{2} \cdots u_{D}$, 其 出边邻居为所有标识为 $v=u_{2} u_{3} \cdots u_{D} q_{1} \cdots q_{m}(0 \leqslant$ $m \leqslant 2$, 当 $m=0$ 时, 形式为 $q_{1} \cdots q_{m}$ 的串为空串) 的节点.
FissionE 中的路由过程与 Kautz 图中的路由过 程基本相同, 图 11 给出了从节点 012 到节点 102 的 路由过程 $(012->121->21->102)$. FissionE 使 用 Kautz_hash 命名算法为每个资源生成 100 位的 Kautz 串作为其标识. 资源被发布到节点标识为其 标识前缀的唯一节点上.

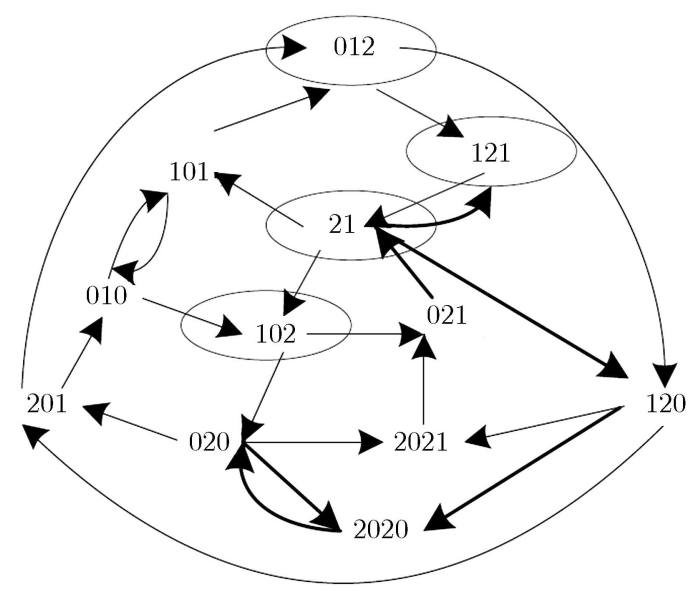

图 11 FissionE 拓扑与路由 ${ }^{[20]}$

FissionE 只能支持以 2 为基的 Kautz 图, 其入 度为 2 , 平均度数为 4 . 因此, 在网络带宽支持高节点 度数的情况下, FissionE 无法采用增加节点度数的方 式提高系统性能.

\subsection{Moore}

为了设计一种能够支持以任意 Kautz 图进行构 建的, 支持任意节点数的高效 P2P 网络, 文献 [21] 提 出了 Moore. 为了达到该目标, Moore 通过在 Kautz 图中应用 PLG 技术 ${ }^{[22]}$ 提出了不完全 Kautz 图 (incomplete Kautz digraph). 此外, 为了选择理想的 边集, 以在构建不完全 Kautz 图的过程中使连通度 (connectivity) 尽量接近 $d$, Moore 应用了 Kautz 因 式分解 (Kautz factorization) 技术 ${ }^{[23]}$.

由于PLG技术和Kautz因式分解均提出于多处 理器可扩展互连网络设计领域, 因此我们认为, 以下 两个原因使得不完全Kautz图不能应用于P2P网络.

首先, PLG 技术和 Kautz 因式分解都是集中式 算法, 无法在全分布的 $\mathrm{P} 2 \mathrm{P}$ 环境下实现. 例如, 当一 个新节点加入 Moore 时, 系统需要知道目前是哪一 个因式 (factor) 正在使用, 以及该因式的哪些节点已 经被选择, 这些信息等价于当前系统的全局拓扑信 
息.

其次, 在某些临界状态, 系统中所有节点的标识 将发生改变. 例如, 假设一个 Moore 系统初始化于 Kautz 图 $K(2,1)$. 当系统节点数达到 6 时, 所有节点 的标识长度都将由 2 变为 3 . 这种变化每当系统节 点数达到 $d^{D}+d^{D-1}$ (例如 $2^{20}+2^{19}=1.5 \mathrm{M}$ ) 时都将 发生, 显然在大规模 $\mathrm{P} 2 \mathrm{P}$ 系统中是不可行的.

\section{6 总结}

本文借鉴线图技术中边点变换的思想, 针对
Kautz 图的特点提出了 D-Kautz 图技术. 使用 DKautz 图技术, 我们进而提出了一种新颖的 P2P 网 络—SKY, SKY 是第一个能够基于以任意大于 1 的正整数为基的 Kautz 图进行构建的 P2P 网络.

我们注意到线图技术能够应用于多种不同的常 量度数拓扑图. 因此, 我们计划在未来工作中把边点 变换的思想应用于其他拓扑图, 例如蝴蝶 (butterfly) 拓扑等. 我们将研究 D-Kautz 图技术的一般化 (generalization) 方法, 以得到一种更加通用的, 能够适用 于多种常量度数 P2P 网络的拓扑维护技术.

\section{参考文献}

1 Li D S, Cao J N, Chan K C C, et al. Delay-bounded range queries in DHT-based peer-to-peer systems. In: ICDCS 2006. Lisboa: IEEE Press, 2006. 64

2 Zhang Y M, Li D S, Chu R, et al. PIBUS: a network memory-based peer-to-peer IO buffering service. In: Networking 2007. LNCS, Vol 4479. Atlanta: Springer-Verlag, 2007. 1237-1240

3 张一鸣, 李东升, 卢锡城. 虚拟计算环境中的可扩展分布式资源信息服务. 软件学报, 2007, 18(8): 1933-1942

4 Dabek F, Kaashoek M F, Karger D, et al. Wide-area cooperative storage with CFS. In: SOSP 2001. Banff: ACM Press, 2001. 202-215

5 Cox R, Muthitacharoen A, Morris R T. Serving DNS using a peer-to-peer lookup service. In: IPTPS 2002 . LNCS, Vol 2429. Cambridge: Springer-Verlag, 2002. 155-165

6 Annapureddy S, Freedman M J, Mazières D. Shark: scaling file servers via cooperative caching. In: NSDI 2005. Boston: USENIX Press, 2005. 129-142

7 Stoica I, Morris R, Karger D R, et al. Chord: a scalable peer-to-peer lookup service for Internet applications. IEEE ACM Trans Netw, 2003, 11(1): 17-32

8 Rowstron A, Druschel P. Pastry: scalable, decentralized object location, and routing for large-scale peer-to-peer systems. In: Middleware 2001. LNCS, Vol 2218. Heidelberg: Springer-Verlag, 2001. 329-350

9 Zhao B Y, Huang L, Stribling J. Tapestry: a resilient global-scale overlay for service deployment. IEEE J Sel Area Comm, 2004, 22(1): 41-53

10 Maymounkov P, Mazieres D. Kademlia: a peer-to-peer information system based on the xor metric. In: IPTPS. LNCS, Vol 2429. Cambridge: Springer-Verlag, 2002. 53-65

11 Harvey N J A, Jones M B, Saroiu S, et al. Skipnet: a scalable overlay network with practical locality properties. In: USITS 2003. Seattle: USENIX Press, 2003. 113-126

12 Ratnasamy S, Francis P, Handley M, et al. A scalable content addressable network. In: SIGCOMM 2001. San Diego: ACM Press, 2001. 161-172

13 Malkhi D, Naor M, Ratajczak D. Viceroy: a scalable and dynamic emulation of the butterfly. In: PODC 2002. Monterey: ACM Press, 2002. 183-192

14 Kumar A, Merugu S, Xu J J, et al. Ulysses: a robust, low-diameter, low-latency peer-to-peer network. In: ICNP 2003. Atlanta: IEEE Press, 2003. 258-267

15 Shen H, Xu C, Chen G. Cycloid: a scalable constant-degree P2P overlay network. Perf Eval, 2005, 63(3): 195-216

16 Fraigniaud P, Gauron P. D2B: a de Bruijn based content-addressable network. Theor Comput Sci, 2006, 355(1): 65-79

17 Kaashoek F, Karger D. Koorde: a simple degree-optimal distributed hash table. In: IPTPS 2003. LNCS, Vol 2735. Berkeley: Springer-Verlag, 2003. 98-107

18 Loguinov D, Kumar A, Rai V, et al. Graph-theoretic analysis of structured peer-to-peer systems: routing distances and fault resilience. In: SIGCOMM 2003. Karlsruhe: ACM Press, 2003. 395-406 
19 Gai A T, Viennot L. Broose: a practical distributed hash table based on the de Bruijn topology. In: International Conference on Peer-to-Peer Computing 2004. Switzerland: IEEE Press, 2004. 167-174

20 Li D S, Lu X C. A novel constant degree and constant congestion DHT scheme for peer-to-peer networks. Sci China Ser F-Inf Sci, 2005, 48(4): 421-436

21 Guo D K, Wu J, Chen H H, et al. Moore: an extendable peer-to-peer network based on incomplete Kautz digraph with constant degree. In: INFOCOM 2007. Anchorage: IEEE Press, 2007. 821-829

22 Fiol M A, Llado A S. The partial line digraph technique in the design of large interconnection networks. IEEE Trans Comput, 1992, C-41(7): 848-857

23 Tvrdik P. Factoring and Scaling Kautz Digraphs. Technical Report LIP ENSL 94-15. 1994

$24 \mathrm{Lu} \mathrm{X} \mathrm{C,} \mathrm{Wang} \mathrm{H} \mathrm{M,} \mathrm{Wang} \mathrm{J.} \mathrm{Internet-based} \mathrm{virtual} \mathrm{computing} \mathrm{environment} \mathrm{(iVCE):} \mathrm{concepts} \mathrm{and} \mathrm{architecture.} \mathrm{Sci} \mathrm{China}$ Ser F-Inf Sci, 2006, 49(6): 681-701

\section{附录 A}

在该附录中我们将简要分析简单的前缀或后缀匹配策略 (prefix/suffix matching policy) 不能应用于 SKY 系统的原 因.

所谓前缀 (或后缀) 匹配策略是指资源 $O$ (对应 Kautz 串为 $s$ ) 应该发布到节点 $v$, 当且仅当 $v$ 是 $s$ 的一个前缀 (或 后缀) 字符串.

首先假设 SKY 中采用前缀匹配策略. 根据定理 1 , 容易 推出在变换 $G^{\prime}=\mathrm{D}-\operatorname{Kautz}(G, v)$ 后, 图 $G^{\prime}$ 中的新节点形为 $\alpha v_{1} v_{2} \cdots v_{m}$, 其中 $\alpha \in\{0,1,2, \ldots, d\}, \alpha \neq v_{1}$. 显然在这种
情况下节点 $v$ 上的资源可能将找不到合适的符合前缀匹配策 略的负责点.

然后考虑 SKY 中采用后缀匹配策略的情况. 在这种策 略下将没有一种分布式算法能够保证消息将最终到达唯一的 负责节点. 假设消息的目的 Kautz 串为 $s=s_{1} s_{2} \cdots s_{r}$. 我 们将无法得知在目前的 SKY 系统中存在 $s$ 的哪一个后缀, 也就是说, 负责节点可能为

$s_{r}, \quad s_{r-1} s_{r}, \quad s_{r-2} s_{r-1} s_{r}, \quad \ldots, \quad s_{1} s_{2} \cdots s_{r}$.

路由消息必须保证下一跳节点能够匹配 $s$ 的更长的后 缀. 然而从定理 1 容易看出 D-Kautz 图显然不支持这种要 求. 